\title{
El libro y las editoriales académicas según los investigadores brasileños de Ciencias Sociales y Humanidades
}

\section{Books and academic editorials according to Brazilian researchers in Social Sciences and Humanities}

\author{
Elea Giménez-Toledo; Carlos-Miguel Tejada-Artigas; Aline Borges-De-Oliveira
}

Cómo citar este artículo:

Giménez-Toledo, Elea; Tejada-Artigas, Carlos-Miguel; Borges-De-Oliveira, Aline (2019). "El libro y las editoriales académicas según los investigadores brasileños de Ciencias Sociales y Humanidades". El profesional de la información, v. 28, n. 6, e280603.

https://doi.org/10.3145/epi.2019.nov.03

Artículo recibido el 15-07-2019

Aceptación definitiva: 11-10-2019

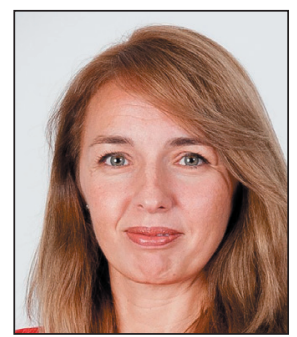

Elea Giménez-Toledo

https://orcid.org/0000-0001-5425-0003

Consejo Superior de Investigaciones Científicas

Centro de Ciencias Humanas y Sociales Albasanz, 26-28. 28037 Madrid, España elea.gimenez@cchs.csic.es

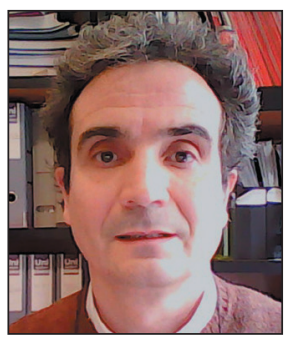

Carlos-Miguel Tejada-Artigas $\bowtie$ https://orcid.org/0000-0002-2767-5636

Universidad Complutense de Madrid Facultad de Ciencias de la

Documentación

Santísima Trinidad, 37. 28010 Madrid, España cmtejada@ucm.es

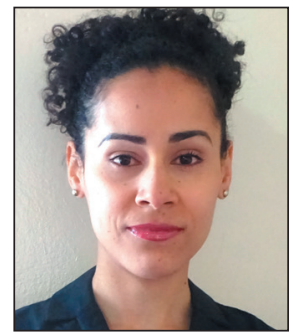

Aline Borges-De-Oliveira

https://orcid.org/0000-0001-5877-3290

alineoliveira090@gmail.com

\section{Resumen}

Este estudio pretende conocer los hábitos y las valoraciones de los investigadores en Ciencias Sociales y Humanidades de Brasil en torno a las publicaciones científicas y en especial sobre los libros académicos. Son varios los aspectos que se tratan: sus hábitos de publicación; sus opiniones sobre el formato libro como medio de comunicación, y sus valoraciones sobre las editoriales académicas basadas en su experiencia. Otros asuntos concretos de las monografías que se abordan son el pago por publicar, las editoriales universitarias y el acceso abierto. La investigación se ha basado en un cuestionario remitido a los coordinadores brasileños de los grupos de investigación de las Ciencias Sociales y Humanidades (16.542 investigadores) y obtuvo una tasa de respuesta del 12\% (1.977 investigadores). El análisis de las respuestas recibidas destaca el valor que dan estos académicos al libro como publicación científica e identifica las debilidades del sector académico brasileño: falta de transparencia en procesos editoriales, financiación, y distribución. En la determinación del prestigio de las editoriales, los investigadores valoran la calidad de las obras publicadas, su transparencia y su rigor en los procesos editoriales.

\section{Palabras clave}

Evaluación de libros; Libros académicos; Editoriales académicas; Indicadores de calidad; Investigadores; Humanidades y Ciencias Sociales; Evaluación científica; Publicación académica; Comunicación académica; Brasil.

Financiación

Proyecto Mineco/Feder CSO2015-63693-P. Las prensas universitarias iberoamericanas y el libro científico en español: calidad, modelos de negocio y política institucional; Capes (Brasil). 


\begin{abstract}
This study aims to know the habits and assessments of researchers in Social Sciences and Humanities of Brazil in relation to scientific publications and especially on academic books. Thus, there are several aspects that are addressed: their publishing habits; their opinions on the book format as a means of communication, and their assessments of academic publishers based on their experience. Other specific issues of the monographs that are addressed are the payment to be published, university publishers, and open access. In this case, the data compilation has been based on a survey sent to Brazilian coordinators of Social Sciences and Humanities research groups (16,542 researchers). The response rate was of $12 \%$ (1,977 researchers). The analysis of the answers received stand out the value that these academics grant to the book as a scientific publication and identifies the weaknesses of the Brazilian academic sector: the lack of transparency in editorial processes, financing, and distribution. In certain cases, the prestige of publishers, and their rigor and transparency were positively evaluated.
\end{abstract}

\title{
Keywords
}

Book assessment; Academic book publishers; Academic monographs; Quality indicators; Researchers; Humanities and Social Sciences; Scientific assessment; Scholarly publishing; Scholarly communication; Brazil.

\section{Introducción}

Los libros son uno de los principales medios de comunicación científica en las Humanidades y en buena parte de las Ciencias Sociales. Su importancia se ha puesto de manifiesto en estudios de producción científica, en análisis de citas y en las propias cifras del mercado editorial (Broadus, 1971; Nederhof, 2006; Giménez-Toledo; Román-Román, 2009; Ardanuy-Baró, 2009; Giménez-Toledo, 2017). En algunos de estos trabajos se observa cómo los investigadores de estos campos publican en una amplia variedad de medios, aunque tienen una especial predilección por los libros publicados en su lengua vernácula y en editoriales nacionales (Engels; Ossenblok; Spruyt, 2012; Sivertsen, 2014; Kulczycki et al., 2018; Kulczycki, 2018).

Los libros son uno de los principales medios de comunicación científica en las Humanidades y en buena parte de las Ciencias Sociales

Por otra parte, la percepción de la calidad o relevancia de las editoriales ha sido también objeto de estudio en algunas ocasiones (Giménez-Toledo; Tejada-Artigas; Mañana-Rodríguez, 2013), y desde luego, forma parte del trabajo que se realiza en procesos de evaluación en países europeos (Giménez-Toledo et al., 2019; Mañana-Rodríguez; Pölönen, 2018).

En el caso específico de Brasil no se han localizado estudios previos que traten la percepción del libro y las editoriales por parte de investigadores brasileños en Ciencias Sociales y Humanidades (en adelante, CSH). Sí que se han localizado y analizado trabajos que analizan la importancia de los libros científicos en la evaluación de los programas de posgrado que realiza Capes (Coordenação de Aperfeiçoamento de Pessoal de Nível Superior) de forma general (Miranda; Mugnaini, 2014; Rodrigues et al., 2018) o de áreas científicas concretas como Psicología (Menandro et al., 2011). Las editoriales universitarias brasileñas también han sido objeto de algunos trabajos (Bufrem, 2015; Marques-Neto; Rosa, 2010; Paolinelli; Gaydeczka; Anjo, 2019).

La realización de este estudio se inscribe en una serie de análisis que se están realizando sobre la edición académica iberoamericana y sus protagonistas -autores, editores, evaluadores, etc.- que permiten disponer de un conocimiento más preciso sobre este eje central de la comunicación científica en CSH. Además permiten destacar la producción de libros académicos en la región, tan abundante, desigual, y poco visible y reconocida. De este modo no sólo se hace hincapié en la relevancia del libro sino también en el propio sector editorial del libro académico iberoamericano, que además de ser una potente industria cultural, contribuye a la diversidad de contenidos científicos y al plurilingüismo en la comunicación científica ${ }^{1}$.

\section{Objetivos}

Este estudio se centra en la relación de los académicos con los libros y tiene como objetivos concretos:

- identificar los hábitos de publicación de los investigadores brasileños en disciplinas de Humanidades y Ciencias Sociales;

- conocer sus valoraciones acerca del libro académico como medio de comunicación y fuente de información;

- identificar las características que determinan la calidad de una editorial académica, a juicio de los propios investigadores;

- examinar las experiencias de los autores sobre los procesos de revisión de sus originales;

- realizar una primera aproximación sobre las prácticas de pago por publicación;

- conocer su percepción sobre las editoriales universitarias y sobre la evolución que debe tener el acceso abierto en el campo de las monografías.

\section{Metodología}

Este trabajo está basado en la realización de una encuesta² dirigida a los directores brasileños de los grupos de investigación de CSH, como muestra de la comunidad de investigadores brasileños en estas disciplinas. Se tomó en consideración 
el Diretório dos grupos de pesquisa no Brasil del Conselho Nacional de Desenvolvimento Científico e Tecnológico (CNPq), localizándose 19.746 directores, de los cuales 2.198 estaban duplicados, ya que un mismo académico puede dirigir varios grupos de investigación.

http://lattes.cnpq.br/web/dgp

Se diseñó un cuestionario similar al utilizado en el estudio de percepción de calidad de editoriales realizado en España (Giménez-Toledo; Tejada-Artigas; Mañana-Rodríguez, 2013), aunque se adaptó al contexto brasileño.

La invitación para cumplimentar el cuestionario fue remitida a 16.542 académicos, directores de grupos de investigación del CNPq con direcciones de correo electrónico activas. El cuestionario online fue cumplimentado a lo largo del último trimestre de 2017, obteniéndose un total de 1.977 respuestas, lo que supone una tasa de respuesta del $12 \%$. El procesamiento de los datos estadísticos se realizó con el software SPSS v.25.

\section{Resultados}

Los resultados obtenidos se presentan siguiendo el orden de las preguntas en el cuestionario:

- perfil del investigador;

- hábitos de publicación y opiniones sobre la importancia del libro académico;

- calidad de las editoriales;

- procesos de evaluación de originales aplicados en las editoriales;

- pago por publicación;

- opinión sobre las editoriales universitarias;

- acceso abierto.

\subsection{Perfil del investigador}

\subsubsection{Categoría profesional}

El cuestionario recogía una primera pregunta sobre la categoría profesional de los académicos. Como era de esperar, al ser éstos directores de grupos de investigación, la mayoría son de las clases de profesores universitarios de más categoría en Brasil: adjuntos, asociados y titulares, tal como podemos observar en el gráfico 1 , que recoge todas las respuestas.

\subsubsection{Género}

Las respuestas se distribuyeron de forma muy equilibrada: $51,9 \%$ (1.003 casos) de quienes respondieron fueron hombres, mientras que el $48,1 \%$ (929) fueron mujeres.

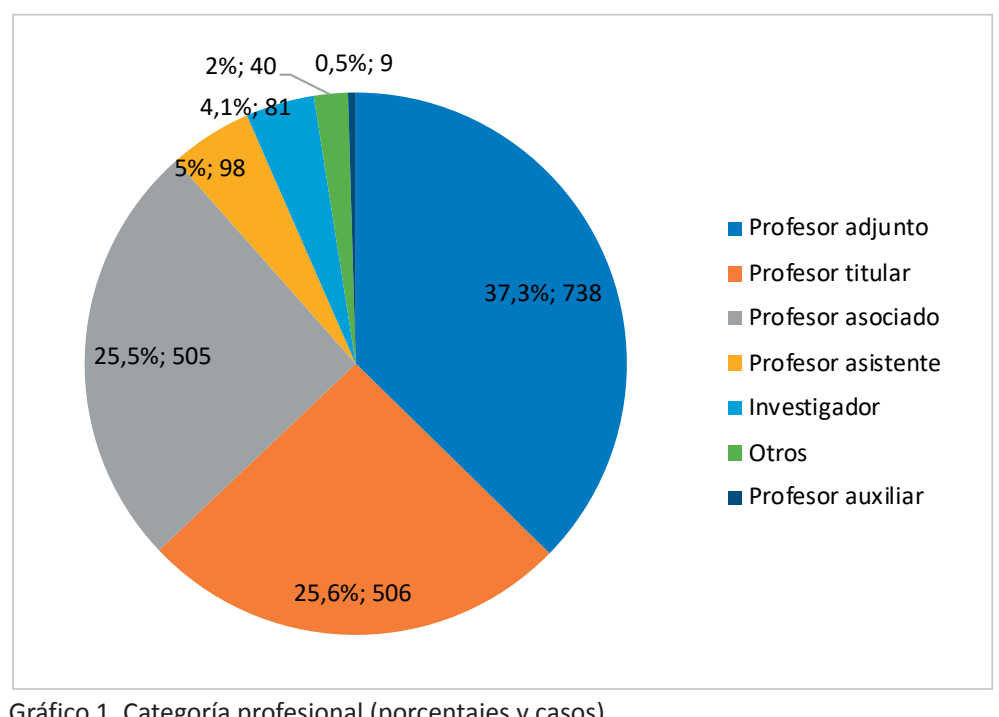

\subsubsection{Edad}

El 83,7\% (1.649 casos) de quienes respondieron a la pregunta tiene más de 40 años. Los de 40 años o menos son el 16,2\% (320) (tabla 1).

\subsection{4. Área de conocimiento}

La pregunta en la que los investigadores debían señalar sus áreas de conocimiento era multirespuesta, ya que puede haber multidisciplinariedad entre los encuestados. Las áreas más señaladas han sido:

- Educación (490 respuestas, 24,8\% de los 1.977 casos que respondieron al cuestionario);

- Filología/Lingüística (287 respuestas, 14,5\%);

- Administración pública y de empresas, contabilidad y turismo (240 respuestas, 12,1\%).

A continuación, figuran las áreas:

- Comunicación e información (187 respuestas, 9,5\%);

- Historia (185 respuestas, 9,5\%);

- Sociología (186 respuestas, 9,4\%);

- Derecho (181 respuestas, 9,2\%);

- Psicología (157 respuestas, 7,9\%);

- Filosofía (134 respuestas, 6,8).
Tabla 1. Edades de los directores de grupos de investigación

\begin{tabular}{|l|c|c|}
\hline \multicolumn{1}{|c|}{ Edad } & n & \% \\
\hline$<30$ & 26 & 1,3 \\
\hline $31-40$ & 294 & 14,9 \\
\hline $41-50$ & 597 & 30,5 \\
\hline $51-60$ & 689 & 35,0 \\
\hline$>61$ & 363 & 18,4 \\
\hline
\end{tabular}




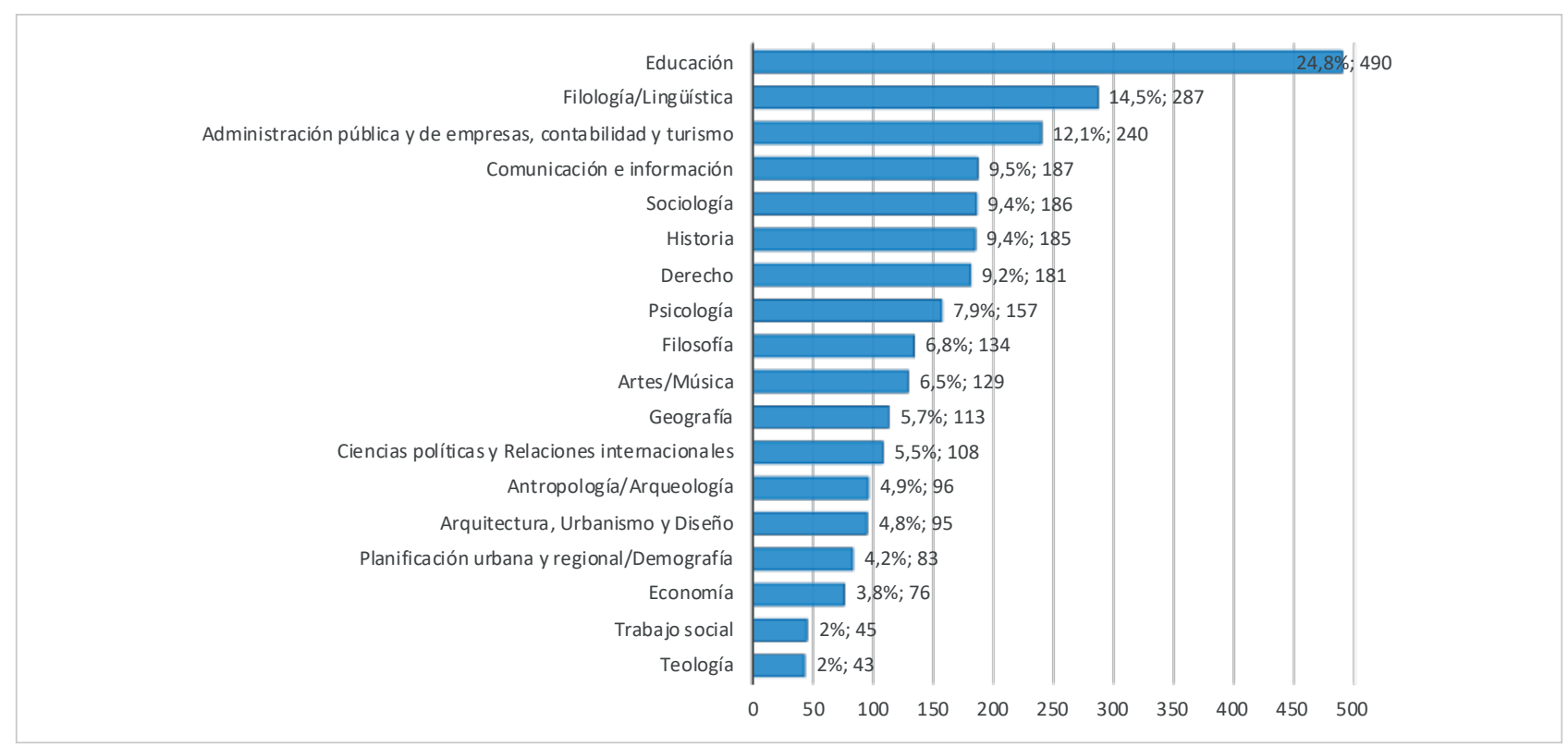

Gráfico 2. Áreas de conocimiento de los investigadores (porcentajes y casos).

Además, hay que destacar que hubo respuesta por parte de investigadores de todas las áreas de las CSH de Brasil (véase el gráfico 2).

\subsubsection{Adscripción institucional}

Al cuestionario respondieron académicos de 279 organismos, la mayoría universidades federales, entre las que destacan:

- Universidade Federal Fluminense (UFF), 60 profesores, 3\%;

- Universidade Estadual Paulista Júlio de Mesquita Filho (Unesp), 56 profesores, 2,8\%;

- Universidade Federal do Rio Grande do Sul (Ufrgs), 55 profesores, 2,8\%;

- Universidade Federal do Rio de Janeiro (UFRJ), 53 profesores, 2,7\%;

- Universidade Federal de Minas Gerais (UFMG), 45 profesores, 2,3\%;

- Universidade de São Paulo (USP), 44 profesores, 2,2\%.

\subsection{Hábitos de publicación de los inves- tigadores y opiniones sobre el libro aca- démico}

\subsubsection{Tipo de publicación para difundir re- sultados de investigación}

Se pidió a los investigadores que asignaran una puntuación entre 0 y 5 a los diferentes tipos documentales en función de su importancia para la difusión de sus resultados de investigación. El artículo de revista arbitrada obtuvo la media más alta de las respuestas, un 4,52, pero hay que destacar que tanto el formato libro como el de capítulo de libro también obtuvieron unas medias muy altas (4 para los libros y 3,71 para los capítulos).

\subsubsection{Bibliografía utilizada}

De forma complementaria, se solicitó a los académicos que puntuaran los anteriores tipos documentales, no como canal de difusión de resultados sino como fuente de información para sus propias investigaciones. Como era previsible, hubo una fuerte correspondencia de las respuestas con las obtenidas en la cuestión anterior. El artículo de revista arbitrada obtuvo una media de 4,55 , seguida del formato libro con 4,29, y del capítulo de libro con 4,06.

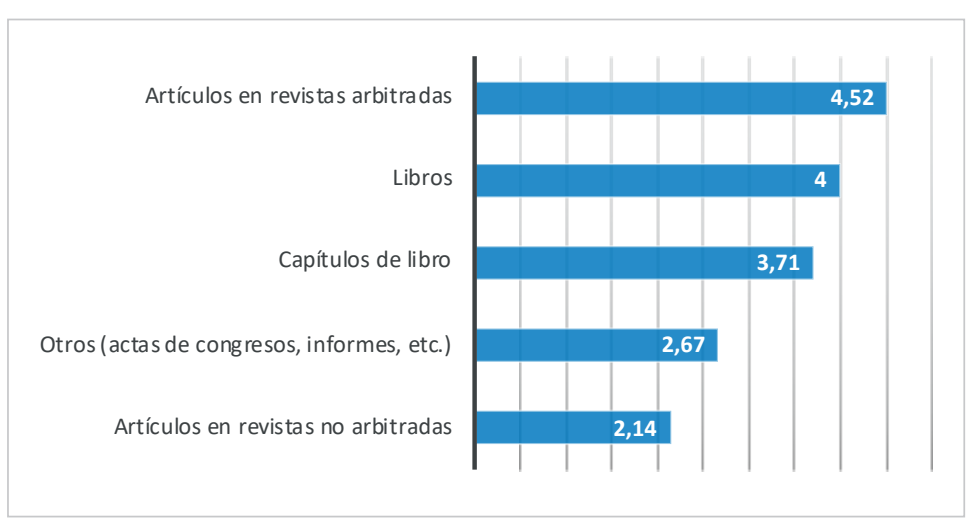

Gráfico 3. Tipos de publicaciones más relevantes o prioritarias para difundir los resultados de la investigación

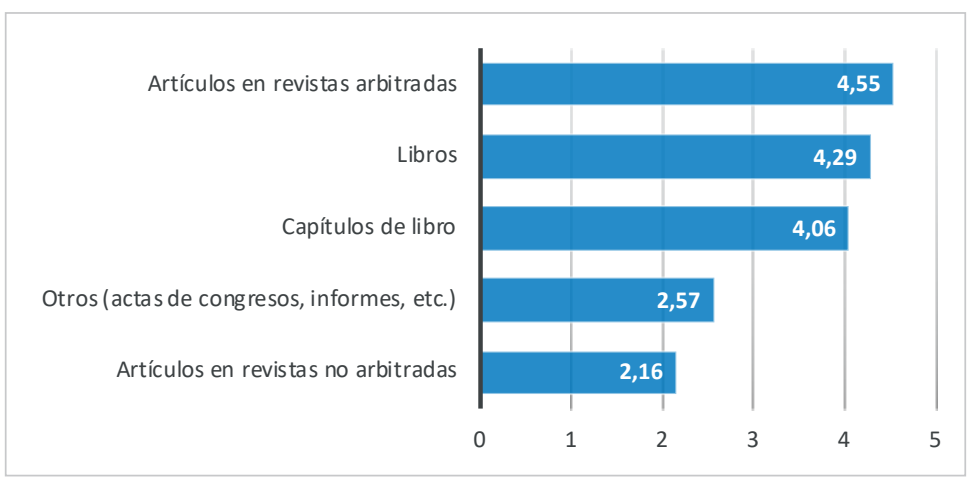

Gráfico 4. Tipos de documento en la bibliografía que preferentemente utilizan los investigadores 


\subsubsection{Origen geográfico de las fuentes bibliográficas}

De una forma mayoritaria (un 70,6\%, 1.395 casos), los investigadores señalan documentos de Brasil. Además, un 49,7\% (982) señala que emplea documentación de Iberoamérica, el 43,4\% (859) del área anglosajona y el 35,4\% (699) de Europa continental.

\subsubsection{El libro en el entorno académico}

El cuestionario pretendía también recabar la opinión de los investigadores sobre la importancia del libro como publicación científica, el futuro del libro electrónico y el estado de los libros didácticos (manuales universitarios). Al igual que en preguntas anteriores, los investigadores debían puntuar de 0 a 5 distintas afirmaciones sobre estos tres asuntos.

La primera afirmación, "el formato libro, por su mayor extensión, es más adecuado para la difusión científica de mis investigaciones", consiguió una media de 3,63 lo que indica una gran aceptación. También la siguiente aseveración, "el libro formato electrónico será más importante que el formato papel para los libros académicos en los próximos dos años", tuvo un grado de aceptación muy alto, con una media de 3,46. La tercera afirmación, "el formato libro es cada vez menos necesario en la docencia universitaria", con una media obtenida de tan sólo un 1,92, demuestra que el libro sigue siendo muy importante en la enseñanza universitaria en Brasil.

Resultan especialmente interesantes las razones aportadas por los investigadores acerca de las tres cuestiones abordadas. A continuación, se resumen las más destacadas.

\subsubsection{El libro como canal de comunicación de resultados de investigación}

Los comentarios recogidos sobre este aspecto se pueden agrupar en tres líneas:

- la importancia del libro académico;

- el libro frente al artículo de revista;

- la escasa valoración del libro en la evaluación de los investigadores.

Muchos investigadores recalcan la importancia del libro en el área de las CSH porque permite la difusión del conocimiento de una manera más extensa, indicando como ejemplo cuatro tipos de investigaciones:

- reflexiones teóricas, que permiten reflejar el pensamiento de una forma más elaborada y profunda;

- contribuciones de las áreas que trabajan con imágenes, como Artes, o Planificación Urbana Regional;

- libros didácticos, que son imprescindibles para conocer el estado de la cuestión de un determinado campo científico;

- investigaciones cualitativas.

Algunas observaciones de los académicos también recogen valoraciones negativas relacionadas con los problemas para poder acceder a ellos:

- deficiente circulación y distribución del libro académico en un país tan grande;

- elevado precio de los ejemplares.

En cuanto al libro frente al artículo de revista, muchos comentarios reflejan que son dos formatos complementarios: los artículos son más adecuados a las novedades de los campos de conocimiento y los libros a las producciones académicas más extensas.

Por último, en lo que respecta a la evaluación de los libros por parte de las agencias, muchos investigadores denuncian su menor valoración frente al artículo de re-
Muchos investigadores recalcan la importancia del libro en el área de las $\mathrm{CSH}$ porque permite la difusión del conocimiento de una manera más extensa 
vista, lo que provoca que al autor sobre todo le interese publicar en revistas. Algunos investigadores incluso llegaron a ligar el futuro del libro académico a la importancia que le otorgue Capes en la evaluación del profesorado, reivindicando que se fortalezca la herramienta Qualis Libros. Se cita el Derecho como ejemplo de área en la que se produce una distorsión entre los patrones reales de comunicación científica y su evaluación. El investigador de este campo no publica fundamentalmente artículos sino libros. Incluso la jurisprudencia se publica en forma de libros. Sin embargo, la evaluación de Capes en el área del Derecho prima la publicación en artículos de revista.

Otros comentarios de los investigadores se refieren a:

- necesidad de que un libro pase por un proceso de evaluación similar al del artículo;

- requerimiento de que las editoriales sean transparentes en sus procesos de selección de originales;

- diferencia en los procesos editoriales de editoriales universitarias frente a las comerciales, ya que estas últimas suelen publicar con menos controles en la evaluación;

- existencia de grupos de académicos que dominan el mercado editorial brasileño que impiden que los nuevos autores publiquen.

\subsubsection{Observaciones sobre el libro electrónico}

Las respuestas abiertas sobre el libro electrónico giran en torno a tres cuestiones:

- ventajas y desventajas;

- uso en el entorno académico;

- acceso abierto.

En cuanto a las ventajas, los investigadores ven en el formato electrónico una oportunidad para una mayor difusión de los libros, sobre todo de los extranjeros, cuya adquisición en Brasil es muy complicada. También presenta grandes posibilidades para su uso en la enseñanza universitaria. Por lo que respecta a las desventajas, algunos académicos alertan de que, a consecuencia de la facilidad en la producción electrónica, cualquiera puede editar y difundir un libro sin que sea evaluado su contenido. También se advierte de los problemas derivados de la piratería y de que las agencias de evaluación dan preferencia a las ediciones de los libros en papel.

Sobre el uso en el entorno académico, se señala que aún es muy limitado debido al coste del libro electrónico y a los hábitos de trabajo de los investigadores, aún apegados al libro impreso.

Por último, por lo que respecta al acceso abierto, algunos académicos indican la oportunidad que representa destacando el importante trabajo de algunas editoras universitarias que ofrecen versiones gratuitas. Incluso algún encuestado señala que debería existir una ley que obligara a las editoriales a poner sus libros online cuando estuvieran agotados y no tuvieran intención de realizar una nueva edición; también se recomienda que los libros considerados clásicos en la universidad sean publicados en abierto.

Algunas de estas ideas fueron apuntadas por las propias editoriales iberoamericanas en un estudio sobre edición digital y en abierto en la región (Giménez-Toledo; Córdoba-Restrepo, 2018).

\subsubsection{El libro universitario}

Hay varias ideas principales que recogen las observaciones realizadas por los encuestados sobre el libro universitario.

La primera es su importancia primordial en la enseñanza superior ya que por su extensión permite presentar aspectos teóricos y argumentaciones de forma más detallada y global. Esto facilita que el estudiante de grado pueda conocer el estado de la cuestión en una determinada disciplina. Algunos investigadores destacan que cierto conocimiento de algunas áreas solamente está recogido en los libros, como en las Humanidades, la Educación o el Derecho.

Otra idea es la dificultad de los estudiantes para acceder a los libros didácticos por su alto coste. Además, algún investigador indica la falta de fondos de las universidades brasileñas, que se traduce, entre otras cuestiones, en que las bibliotecas no pueden adquirir todos los libros que serían necesarios para tener un catálogo completo y actualizado. Aunque también algún comentario recoge la idea que el libro universitario no será tan utilizado en el medio plazo porque los alumnos cada vez tienden a leer menos libros completos y consultan materiales más sintéticos y reducidos. Este cambio en los hábitos de trabajo de los estudiantes, unido a una falta de reconocimiento del manual docente en los procesos de evaluación, plantea un futuro incierto para los libros didácticos o manuales universitarios y, por tanto, para las editoriales que tradicionalmente los venían publicando.

\subsection{Características de calidad de una editorial académica}

\subsubsection{Razones de elección de editorial para publicar}

El cuestionario incluía una pregunta con opciones de respuesta cerradas (multirrespuesta) para que los investigadores señalasen las razones que motivaron su elección de una determinada editorial para publicar. Se puede observar que hay varios factores que suelen tener en cuenta. Más de la mitad de los investigadores señalaron los siguientes factores:

- calidad de la editorial (74,1\%, 1.464 respuestas); 
- reputación de la editorial $(68,6 \%$, 1.356);

- proceso de evaluación riguroso y transparente $(51,2 \%, 1.013)$.

El gráfico 7 muestra la relevancia que otorgan los investigadores a las distintas cualidades de las editoriales.

Los investigadores también podían indicar en "otros factores", aquellos que no estaban considerados en las respuestas cerradas. La mayoría de estas respuestas abiertas hacen referencia a tres cuestiones:

- importancia de que las editoriales tengan una distribución buena y que participen en eventos científicos para dar a conocer su fondo;

- la editorial esté bien considerada por Qualis/Capes;

- la casa editora cuente con un comité editorial compuesto por profesionales cualificados y expertos en las diferentes áreas de conomiento.

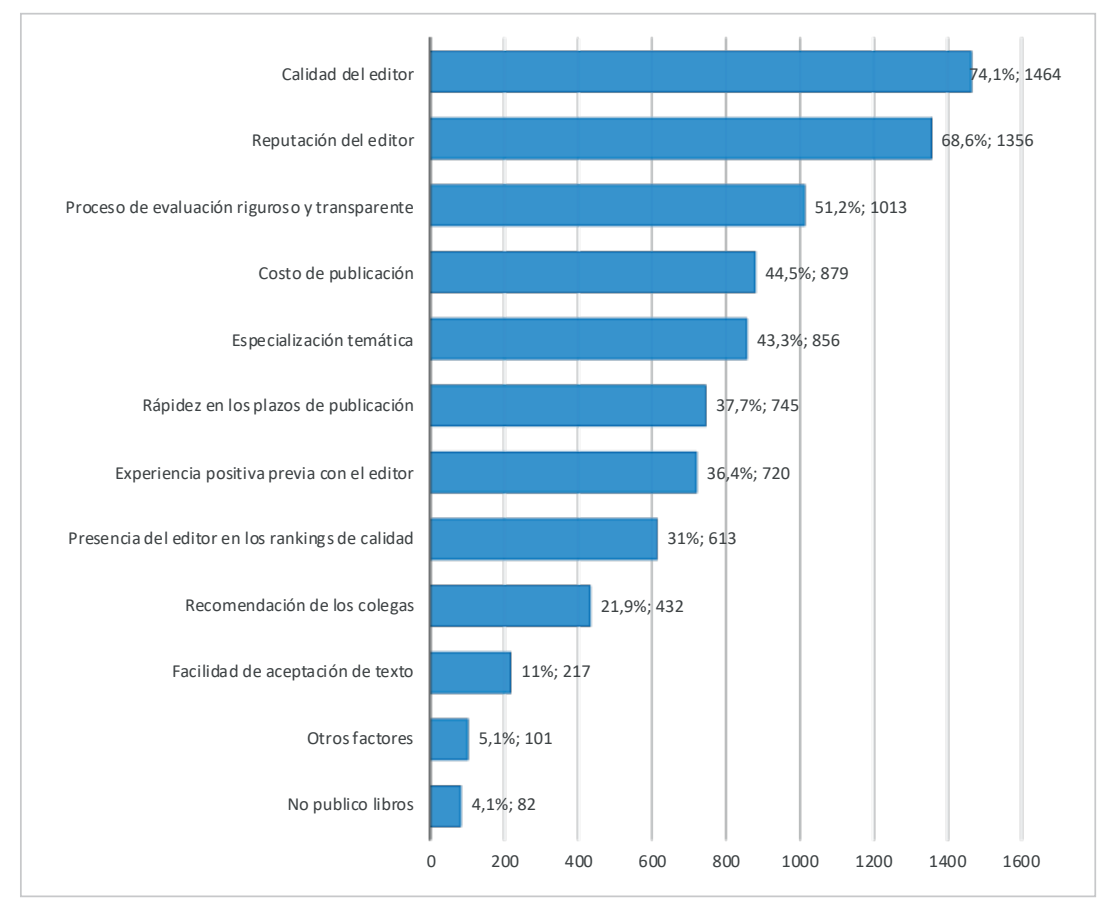

Gráfico 7. Razones de elección de una determinada editorial para publicar un texto (porcentajes y casos)

\subsubsection{Características generales de calidad de una editorial}

Con el objetivo de identificar qué grupo de variables son más importantes a la hora de determinar la calidad general de una editorial, se solicitó a los académicos que puntuaran de 0 a 5 , seis bloques de indicadores propuestos. A partir de las medias obtenidas, se señalan los tres aspectos más importantes:

- calidad de las ediciones de la editorial $(4,47)$;

- sistema de difusión y distribución de la editorial $(4,34)$;

- sistema de evaluación mediante evaluadores de la editorial $(4,24)$.

El gráfico 8 muestra las características generales y las medias de puntuación obtenidas.

\subsubsection{Indicadores de calidad de una editorial}

Una nueva pregunta del cuestionario pretendía conocer los indicadores o características concretas que definen

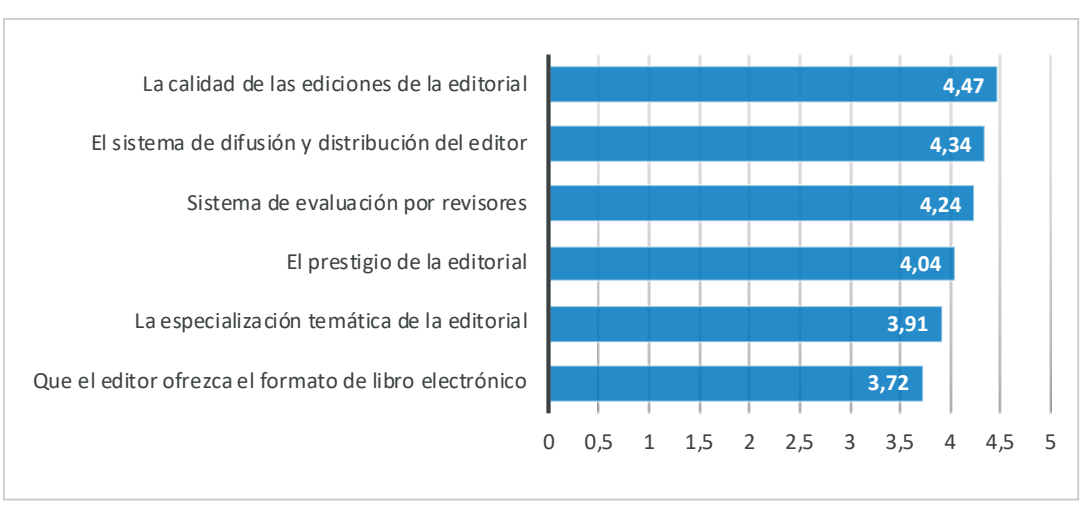

Gráfico 8. Características generales que avalan la calidad de una editorial

la calidad de una editorial. Los que más respuesta han obtenido son los siguientes:

- estructura adecuada (la calidad de las ediciones de la editorial) (1.307 respuestas, 66,1\%);

- revisores externos (1298 respuestas, 65,7\%);

- comités de lectura especializados (internos de la editora) (1.239 respuestas, 62,7\%);

- presencia en librerías especializadas nacionales e internacionales (1.190 respuestas, 60,2\%);

- las agencias de avaluación (Capes y CNPq) evalúan positivamente esta editorial (1.172 respuestas, 59,3\%);

- presentación formal (1.156 respuestas, 58,5\%);

- editoriales con colecciones especializadas (1.030 respuestas, 52,1\%).

En el gráfico 9 pueden observarse todas las características y el peso que les conceden los investigadores.

\subsection{Experiencias de publicación en editoriales académicas}

Uno de los objetivos ya señalados del cuestionario era obtener información de primera mano sobre los procesos de evaluación de originales que llevan a cabo las editoriales académicas. Interesaba que sólo contestaran los académicos que habían tenido una experiencia directa con las editoriales, por lo que en primer lugar tenían que indicar si habían publicado algún libro en alguna editorial, y si esto era así, que respondieran al resto de las preguntas. Como resultado 
1.697 investigadores contestaron a la pregunta, y de ellos el $78,6 \%(1,334)$ respondió de forma afirmativa y el 21,4 (363) de forma negativa.

\subsubsection{Tipo de editoriales en la que han publicado los investiga- dores}

Como continuación a la pregunta anterior, se preguntó -sólo a los académicos que habían publicado- sobre el tipo de editoriales que habían elegido para publicar (pregunta multirrespuesta). Los tres tipos más señalados fueron:

- editorial académica/científica universitaria brasileña (1.023 respuestas, $76,6 \%$ de los que han publicado);

- editorial académica/científica comercial brasileña (743 respuestas, 55,6\%);

- editorial comercial brasileña con colecciones científicas (499 respuestas, 37,4\%\%).

Estos resultados indican que los investigadores brasileños sobre todo publican en editoriales del país y atienden a la importancia de las casas editoras universitarias. El gráfico 10 muestra todas las respuestas y porcentajes obtenidos.

\subsubsection{Forma de contacto entre la editorial y el autor}

Más de la mitad de los investigadores se pusieron en contacto directamente con la editorial para ofrecer su texto para la publicación (931, 69,7\% de los que han publicado); en otros casos, fue el coordinador de una obra colectiva quien contactó con ellos para que escribiesen un capítulo (676; 50,6\%). Esta pregunta era multirrespuesta porque los investigadores pueden haber pasado por distintas situaciones.

\subsubsection{Transparencia de las edito-} riales sobre envío de originales

Por lo que respecta a la transparencia de las editoriales en sus procesos de publicación de originales, 898 investigadores (el $57,7 \%$ de los que contestaron a la pregunta) opinan que las editoriales ofrecen informaciones

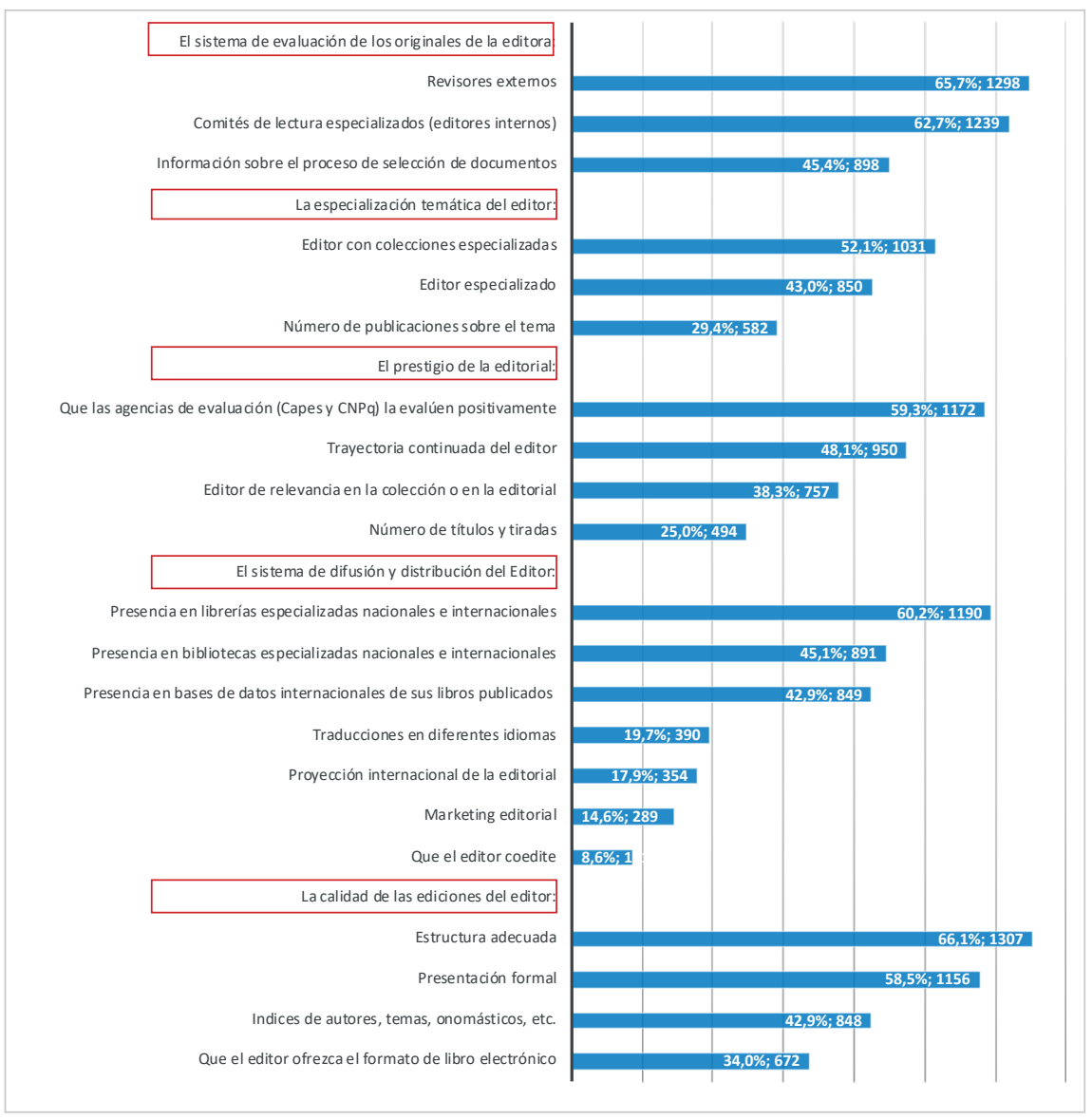

Gráfico 9. Indicadores que representan la calidad de una editorial (porcentajes y casos)

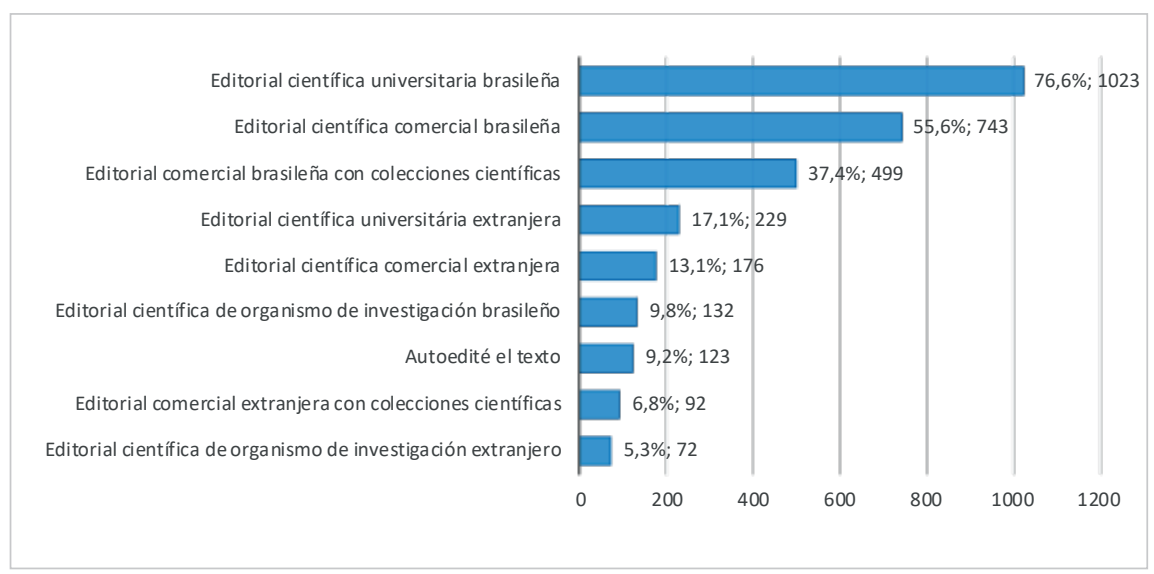

Gráfico 10. Tipo de editorial en la que han publicado los investigadores (porcentajes y casos)

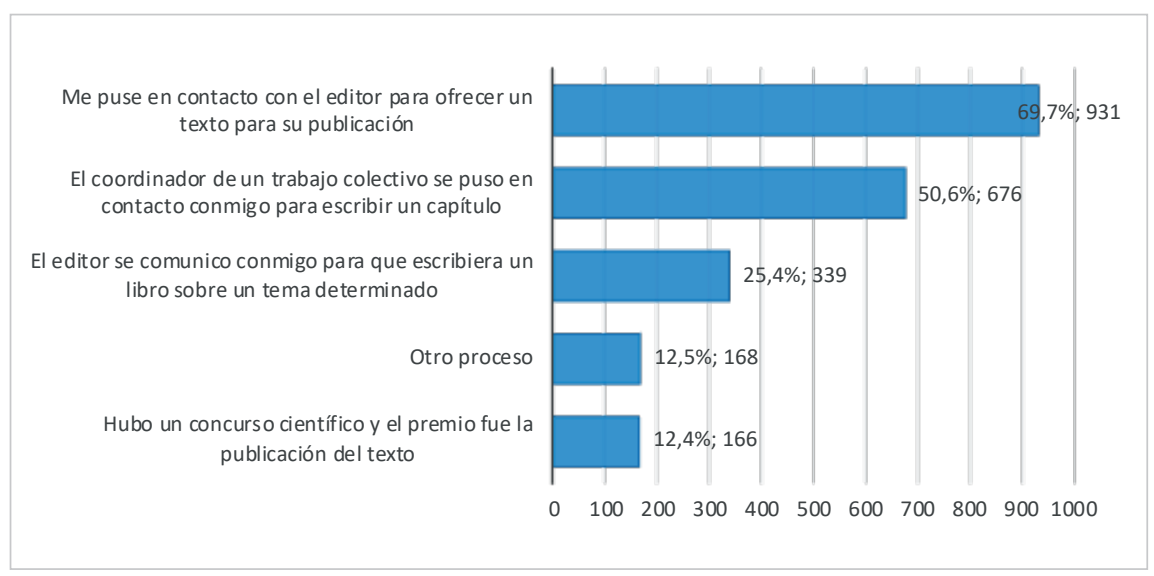

Gráfico 11. Forma de contacto entre la editorial y el autor (porcentajes y casos) 
claras a los autores; mientras que 358 indican que no las ofrecen (23\%); y 299 académicos (19,2\%) no tienen una opinión clara al respecto. Sin duda, como también se desprende de otros estudios previos (Giménez-Toledo, 2017), la transparencia es un aspecto a mejorar en las editoriales.

Entre las respuestas abiertas obtenidas, varias señalan que las editoriales comerciales son las más opacas, mientras que las universitarias suelen ser más transparentes. Algún investigador incluso apunta que cuanto mayor es el prestigio de los editores, menos claro es el proceso, señalando que las editoriales prestigiosas son muy cerradas y publican sólo por invitación y no suelen publicar a investigadores del Norte y del Nordeste de Brasil. También hay varias opiniones que denuncian que hay muchas editoriales comerciales que no están interesadas en la calidad de las obras que publican sino en que la obra sea financiada por el autor.

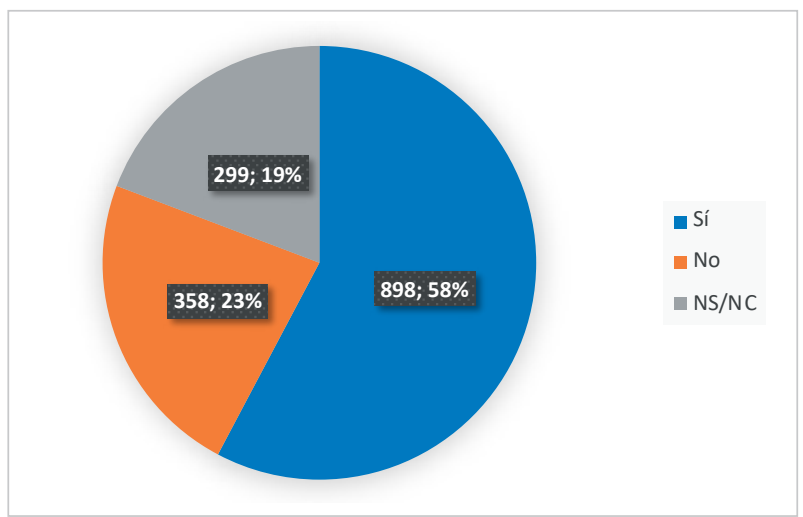

Gráfico 12. Transparencia de las editoriales sobre el envío de originales (casos y porcentajes)

\subsubsection{Transparencia de las editoriales sobre el proceso de selección de originales}

Las percepciones de los investigadores con respecto a la claridad de las editoriales sobre el proceso de evaluación de originales también fueron recogidas en el cuestionario. Es mayor el porcentaje de respuestas positivas al respecto: $50,8 \%$ (775 casos) frente al $26,8 \%$ de respuestas negativas ( 409 casos) y al $22,5 \%$ (343 casos) que no tienen una clara información al respecto. Aunque prácticamente la mitad de los profesores considera que las editoriales son claras en este proceso, el porcentaje tendría que ser mucho mayor, tal como ocurre ya con los editores de revistas científicas que prácticamente la totalidad informa en sus sitios webs al respecto. Como en el caso anterior, cabría esperar que la mayoría de las editoriales ofrecieran información pública sobre la forma de selección de originales.

Precisamente en las respuestas abiertas muchos investigadores denuncian este comportamiento opaco, destacando que o bien nunca recibieron una respuesta de las editoriales, o bien esta respuesta fue muy genérica o ambigua como "el libro está fuera de la política editorial de la empresa", o "el libro no es comercial porque su público es académico". Además, los académicos destacaron que las editoriales sólo señalan los procedimientos de envío, pero casi no dan información ni de los plazos, ni del proceso de arbitraje por pares. Como en la pregunta anterior, también algunas respuestas destacan que las editoriales universitarias son más transparentes que las comerciales, ya que muchas de ellas realizan convocatorias de selección en las que los procesos de evaluación están claros.

\subsubsection{Experiencias de los investigadores en procesos de selección de originales}

Para analizar en detalle los procesos de revisión científica que realizan las editoriales, en primer lugar se preguntó a los profesores si sus propuestas habían pasado por estos procesos. El 58,8\% (910) de los 1.547 investigadores que respondieron a esta pregunta lo hicieron afirmativamente; algunas veces, un 21,1\% (327); un 10,1\% señaló negativamente (157); y un 9,9\% respondió que lo desconocía porque la editorial nunca les dio esta información (153). En el gráfico 13, se pueden observar todas las respuestas.

\subsubsection{Características del proceso de revisión de los ori-} ginales

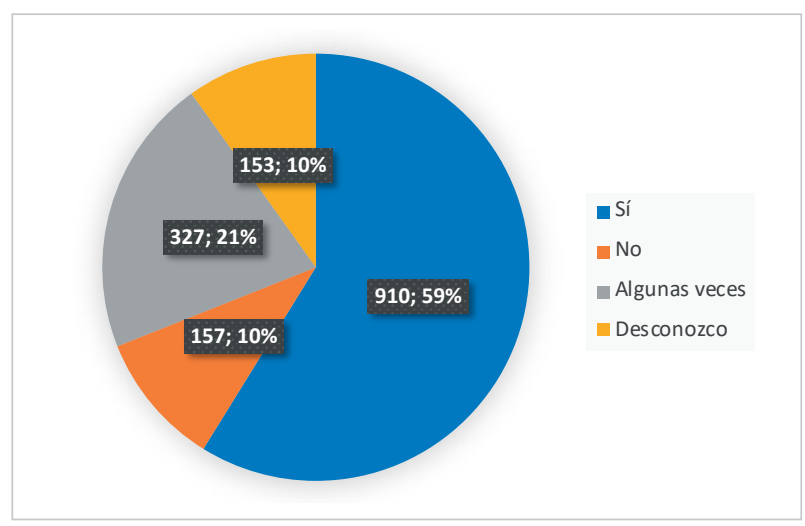

Gráfico 13. Investigadores que han sometido sus libros a procesos de revisión científica de la editorial (casos y porcentajes)

El procedimiento de selección de originales más señalado por los académicos fue la evaluación por un comité de lectura de la propia editorial, con un $43,6 \%$ de las respuestas obtenidas a esta pregunta (787). La evaluación externa a la editorial tuvo una respuesta del 54,3\%, con un sistema de revisión por pares (doble ciego) $(27,3 \%, 493)$, con los informes de evaluadores externos (simple ciego) $(22,8 \%, 411)$ y con un sistema de revisión por pares no anónimo $(4,2 \%, 75)$.

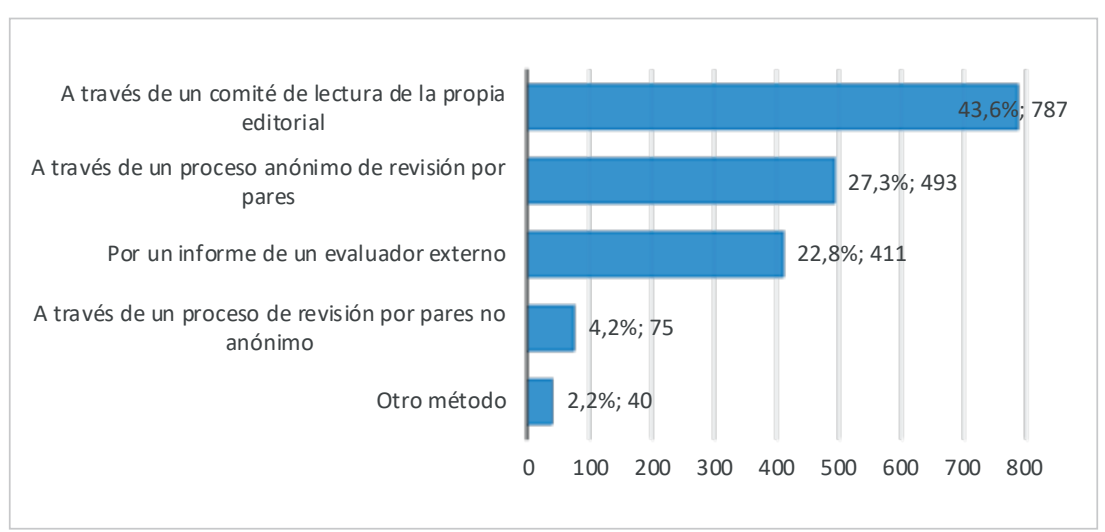

Gráfico 14. Proceso del proceso de revisión científica de los originales (porcentajes y casos). 


\subsubsection{Comunicación motivada del rechazo editorial}

Otro aspecto importante del proceso de selección de los originales es que las editoriales comuniquen razonadamente el resultado de su evaluación. Preguntados por esta cuestión:

- 33\% de los investigadores señaló que sí recibió comunicación motivada (287 casos del total de 871 que respondieron);

- 27,6\% (240) también indicó que sí les proporcionaron esa información, pero con pocos detalles;

- 26,8\% (233) señaló que nunca se la proporcionaron;

- 12,7\% (111) señaló que solamente algunas veces.

De nuevo se observa aquí cómo la práctica editorial pue-

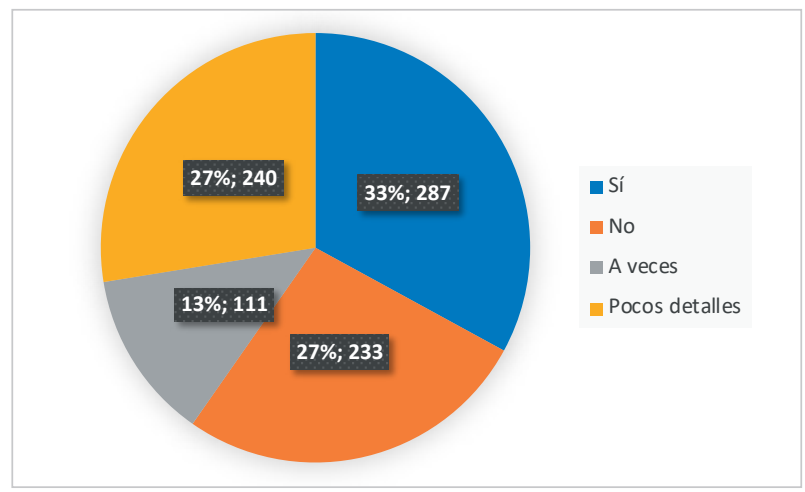

Gráfico 15. Comunicación de las razones científicas de un rechazo (casos y porcentajes) de mejorar en lo que se refiere a la transparencia y comunicación con sus autores.

\subsection{Pago por publicación}

Aunque en un 57,5\% de los casos (902) no se ha pagado por publicar, en un 42,5\% sí se ha hecho, lo que parece un porcentaje bastante alto. Aunque se ha observado en el sector editorial brasileño que la financiación de las obras por parte del autor es un hecho en cierto modo extendido (Giménez-Toledo; Córdoba-Restrepo, 2018), en términos generales es una práctica controvertida que pone en cuestión la calidad de lo que se publica. Por otra parte, este pago por publicación tampoco parece estar directamente vinculado a la publicación de monografías en abierto, lo que justificaría un cierto modelo de negocio.

También resulta llamativo el relativo equilibrio que se produce entre los investigadores que estarían dispuestos a pagar por publicar $(55,4 \%, 1.023)$ y los que no lo harían $44,6 \%, 822)$.

\subsection{Opinión sobre el funcionamiento de las editoriales universitarias y la evolución del acceso abierto}

\subsubsection{Percepción con respecto a las editoriales universitarias de sus instituciones}

Se ofrecían cuatro opciones de respuesta cerradas de las cuales podían elegir solamente una. Más de la mitad de los encuestados, 1.027 (61,9\%) eligieron las opciones "Necesita de mejoras" o "Regular". Entre los que las valoraron de forma positiva, $457(27,6 \%)$ la calificaron de "Buena" y $174(10,5 \%)$ como "Excelente".

Los académicos podían justificar su respuesta en una pregunta abierta. La mayoría de ellos, realizaron críticas negativas, sobre todo indicando la falta de presupuesto de estas casas editoras, aunque también señalaron otros aspectos que atañen a sus políticas de publicación y su distribución. Véase por ejemplo las siguientes respuestas:

"Los editores universitarios no tienen una política muy clara en la aceptación de libros para su publicación".

"Pocas convocatorias para la publicación".

"Falta de divulgación de la editorial".

"Injerencia política, falta de relevancia, falta de distribución".

"No hay regularidad en la política de publicación".

"No es transparente con el autor ni con la selección".

"Tengo la impresión de que la publicación es por indicaciones.

"Debido a que no hay interés comercial, las editoriales universitarias se muestran desinteresadas y lentas".

"Muy cerrada, no permitiendo la entrada de nuevos autores".

"Baja distribución, poca presencia en ferias y librerías"

\subsubsection{Opinión sobre el acceso abierto en las editoriales universitarias}

Uno de los aspectos de las editoriales universitarias de más actualidad es si deben apostar por la publicación en abierto. Sobre esta cuestión se recogió la opinión de los investigadores, que se pronunciaron mayoritariamente a favor, concretamente un $85,5 \%$ de los que respondieron a esta cuestión (1.574). El porcentaje restante, 14,5\% (267) contestó que no está de acuerdo con que las universidades publiquen en acceso abierto.

Como en otras preguntas, los encuestados podían razonar su respuesta. Los comentarios de los partidarios del acceso abierto lo justifican porque estas editoras ya se financian con recursos públicos por lo que deben tener el cometido de contribuir al conocimiento de la sociedad, sobre todo de los alumnos universitarios, que muchas veces no pueden hacer frente a los costes de los libros. Algunos académicos destacan que publicar en abierto permite una mayor difusión de las investigaciones y un aumento de las citas. Otros subrayan el éxito de las editoriales universitarias que ya han asumido el acceso abierto. Por último, algunos comentarios apuntan a que esta tendencia se impondrá en un futuro, incluso algún académico señala que esto se podría producir en cinco años.

Los que se muestran contrarios sobre todo lo hacen por razón del presupuesto. Consideran que las editoriales universitarias deben cobrar para poder tener ingresos y mantenerse, llegando a afirmar que es una utopía creer que las editoriales universitarias pueden vivir únicamente del presupuesto de las universidades. 


\section{Conclusiones y discusión}

Los resultados de este estudio destacan la relevancia del libro académico en las prácticas de publicación y lectura de los investigadores brasileños de CSH. A pesar de que no hay datos globales sobre lo que representan los libros académicos en la producción científica global de Brasil, el patrón de publicación parece coincidir con lo que se ha descrito en estudios europeos (Kulczycki et al. 2018; Giménez-Toledo et al., 2019). Aunque se concede mucha importancia al artículo de revista científica, se pone de manifiesto, como en otros estudios anteriores, que en función del tipo de resultados a publicar resulta más adecuado un canal u otro de comunicación. La agilidad en la publicación y la mejor consideración en los procesos de evaluación son factores adicionales en la buena valoración de los artículos como canales de comunicación científica. Pero evidentemente se reivindica claramente la necesidad de que el libro sea mejor considerado en los procesos de evaluación de la actividad científica. Las agencias de evaluación y financiación de la investigación de distintos países del mundo (Giménez-Toledo; Mañana-Rodríguez; Tejada-Artigas, 2015; Giménez-Toledo et al., 2019) incluyen los libros de investigación, con distintos pesos y consideraciones, en sus procesos de evaluación, y especialmente en las disciplinas de CSH. Es importante destacar la necesidad de contar con procesos de evaluación bien definidos, ya sean de corte cualitativo, ya sean basados en indicadores de libros o editoriales. Esta definición de los procesos de evaluación de libros mejora la transparencia de los procesos de evaluación, genera confianza en los investigadores y permite tomar decisiones más objetivas, no sólo en relación con los investigadores evaluados (Molas-Gallart, 2015) sino también con las editoriales. En cualquier caso, la evaluación de libros pasa por conocer el sector editorial de un país de forma sistemática. En Brasil se localizan estudios de mercado (FIPE, 2017; 2018; Falcó-Parra, 2018) que ayudan a situar el libro académico/científico en el conjunto de la industria editorial, pero no se han localizado estudios que delimiten con concreción los sellos editoriales que publican fundamentalmente libro académico, las cifras de producción de estos sellos individualmente y en conjunto, sus perfiles de especialización o sus indicadores de calidad. La tesis de Borges-De-Oliveira (2018) identifica por primera vez las editoriales brasileñas más prestigiosas, según la opinión de los propios humanistas y científicos sociales brasileños. Contribuye con ello a delimitar aquellas editoriales -tanto universitarias como comerciales- que publican libros relevantes para estas disciplinas científicas.

En este artículo -derivado parcialmente de la tesis- se identifican las características de las editoriales que son importantes para los investigadores en la determinación de la calidad de una editorial. En las preguntas relacionadas con la calidad de las editoriales (motivos para elegirlas, características e indicadores que definen su calidad) se detecta una fuerte correspondencia en las respuestas. La calidad y la reputación de la editorial, unidas al rigor y la transparencia de los procesos editoriales son factores muy relevantes para el conjunto de los investigadores brasileños. Además, esta conclusión coincide con la obtenida en las consultas realizadas entre investigadores españoles (Giménez-Toledo; Tejada-Artigas; Mañana-Rodríguez, 2013).

Los investigadores brasileños de CSH suelen publicar sobre todo en editoriales nacionales y universitarias, hecho que guarda relación con la propia conformación del sector editorial. Las 123 editoriales universitarias que integran $A B E U$, la Asociación Brasileña de Editoriales Universitarias, tienen un gran peso en el conjunto de la edición académica, aunque no puede indicarse con precisión, por la falta de estudios antes apuntada.

En torno a un $42 \%$ de los académicos no tiene una opinión positiva o clara sobre la transparencia de las editoriales acerca del envío de originales y el porcentaje sube al $48 \%$ si se trata del proceso de evaluación. Este aspecto es clave no sólo como una buena y esperada práctica editorial, necesaria para los autores y evaluadores, sino que también es una de las cualidades a las que atienden los sistemas de evaluación de libros en sentido amplio (DOAB o SPI, por ejemplo). https://www.doabooks.org/doab?func=forPublishers\&uilanguage=en\#requirements http://ilia.cchs.csic.es/SPI/revision_originales.html

Estos aspectos también son considerados por los sellos de calidad para libros o colecciones de varios países y regiones (España, Flandes, Finlandia).

http://www.selloceaapq.es

https://www.gprc.be/en/content/what-gprc

https://www.tsv.fi/en/services/label-for-peer-reviewed-scholarly-publications

Esta falta de transparencia se observa también en las cifras sobre la comunicación razonada de un rechazo editorial. La tasa de respuesta es baja para esa pregunta pero además, entre las respuestas que se registran, destaca la falta de comunicación y/o argumentos sobre el rechazo editorial.

La propia experiencia de los autores con las editoriales para publicar sus libros, permite saber que el comité de lectura interno asume habitualmente la tarea de evaluar los textos que se reciben en las editoriales, aunque la evaluación externa es un procedimiento también muy frecuente. De hecho es el más frecuente.

En Brasil la financiación de las obras por parte del autor es un hecho en cierto modo extendido (Giménez-Toledo; Córdoba-Restrepo, 2018), pero no es un esquema especialmente vinculado a la edición de libros en abierto, sino a todo tipo de libros. Eso explica que un 42,5\% de los investigadores indique que ha pagado por publicar. Este porcentaje debe ser entendido en ese contexto aunque, visto desde la perspectiva de otras prácticas editoriales internacionales, hay que indicar la controversia que existe sobre el pago por publicación y la manera en que ese hecho puede afectar a la calidad de lo que se publica. 
Los investigadores brasileños tienen una visión más bien crítica sobre las editoriales de las universidades en las que trabajan. Un 61,9\% indica que necesita mejoras o es regular. Sobre todo aluden a cuestiones de financiación, políticas de publicación y distribución de sus obras. La mayor parte de estos académicos abogan para que estas casas editoras apuesten por el acceso abierto.

A pesar de que la tasa de respuesta es baja, el número de respuestas y la variedad de disciplinas a las que aluden, permiten detectar algunas cuestiones estratégicas y/o recomendaciones:

- Un mejor reconocimiento de las monografías en los procesos de evaluación pasa necesariamente por conocer adecuadamente el sector editorial, disponer de indicadores específicos para editoriales, colecciones y/o libros y, desde luego, porque se produzca un acercamiento entre editoriales y agencias de evaluación de la actividad científica. Asimismo, la transparencia de las editoriales en relación con sus procesos resulta crucial para que los autores y evaluadores dispongan de información que es relevante para ellos. Las casas editoras deben de hacer un esfuerzo en su transparencia ofreciendo informaciones claras sobre sus políticas editoriales y sobre cómo realizan las evaluaciones de los textos, en qué plazos, y sus resultados.

- Las editoriales que publican libros científicos deben cuidar la calidad de sus obras y sus sistemas de selección de originales ya que sus autores y lectores valoran de forma primordial estas características.

- El formato electrónico y el acceso abierto suponen una oportunidad para superar el problema que tiene Brasil en la circulación y distribución de los libros y, en general, para hacer más visible su producción científica. Tanto Metabooks como Scielo Livros permiten avanzar en estos objetivos de manera muy destacada. Sin embargo, las editoriales universitarias apenas están proporcionando metadatos a Metabooks y, por otra parte, la participación de pequeñas editoriales en Scielo Livros está en cierto modo limitada por los costes de acceso. Superar ambas cuestiones requiere estrategias globales por parte del sector editorial o de las instituciones con competencias en ciencia y cultura.

\section{Notas}

1. Iniciativa Helsinki sobre multilingüismo en la comunicación científica https://www.helsinki-initiative.org/es

2. La encuesta se utilizó en la investigación para la tesis doctoral Los libros en Ciencias Sociales y Humanidades en Brasil: un estudio a partir de los investigadores y de las editoriales, auspiciada por la Coordenação de Aperfeiçoamento de Pessoal de Nível Superior (Capes), defendida por Aline Borges-De-Oliveira en la Universidad Complutense de Madrid (Borges-De-Oliveira, 2018).

https://goo.gl/forms/iapuT7QdVQvititL2

\section{Referencias}

Ardanuy-Baró, Jordi (2009). “Els estudis bibliomètrics basats en l’anàlisi de citacions en àrees d’humanitats en el període 1959-2008". BiD: textos universitaris de biblioteconomia i documentació, n. 22.

http://bid.ub.edu/22/ardanuy.htm

Borges-De-Oliveira, Aline (2018). Los libros en Ciencias Sociales y Humanidades en Brasil: un estudio a partir de los investigadores y de las editoriales. Tesis doctoral. Madrid: Universidad Complutense.

https://eprints.ucm.es/55556/1/T41139.pdf

Broadus, Robert N. (1971). "The literature of the social science: a survey of citation studies". International social science journal, n. 23, pp. 236-243.

Bufrem, Leilah-Santiago (2015). Editoras universitárias no Brasil: Uma crítica para a reformulação da prática. São Paulo: EDUSP. ISBN: 9788531415234

Engels, Tim C. E.; Ossenblok, Truyken L. B.; Spruyt, Eric H. J. (2012). “Changing publication patterns in the social sciences and humanities, 2000-2009". Scientometrics, v. 93, n. 2, p. 373-390.

https://doi.org/10.1007/s11192-012-0680-2

Falcó-Parra, Aitor (2018). Estudio de mercado. El mercado editorial en Brasil 2018. São Paulo: ICEX España Exportación e Inversiones.

https://bit.ly/2P5Wxkn

FIPE (2017). Censo do livro digital. São Paulo: Câmara Brasileira do Livro; Sindicato Nacional dos Editores de Libros, SNEL; Fundação Instituto de Pesquisas Econômicas.

https://snel.org.br/wp/wp-content/uploads/2018/02/Apresentacao-Censo-do-Livro-Digital.pdf

FIPE (2018). Produção e vendas do setor editorial brasileiro. São Paulo: Câmara Brasileira do Livro; Sindicato Nacional dos Editores de Libros, SNEL; Fundação Instituto de Pesquisas Econômicas.

https://snel.org.br/wp/wp-content/uploads/2019/04/Apresentacao_pesquisa_ano_base_2018_imprensa.pdf

Giménez-Toledo, Elea (ed.) (2017). La edición académica española. Indicadores y características. Madrid: Federación de Gremios de Editores de España. ISBN: 9788486141660 
http://ilia.cchs.csic.es/SPI/spi-fgee/docs/EAEV2.pdf

Giménez-Toledo, Elea; Córdoba-Restrepo, Juan-Felipe (eds.) (2018). Edición académica y difusión. Libro abierto en Iberoamérica. Bogotá / Granada: Universidad del Rosario / Comares. ISBN: 9789587841664 https://doi.org/10.12804/th9789587841671

Giménez-Toledo, Elea; Mañana-Rodríguez, Jorge; Engels, Tim C. E.; Guns, Raf; Kulczycki, Emanuel; Ochsner, Michael; Pölönen, Janne; Sivertsen, Gunnar; Zuccala, Alesia A. (2019). "Taking scholarly books into account, part II: A comparison of 19 European countries in evaluation and funding". Scientometrics v. 118, n. 1, pp. 233-251.

https://doi.org/10.1007/s11192-018-2956-7

Giménez-Toledo, Elea; Mañana-Rodríguez, Jorge; Tejada-Artigas, Carlos-Miguel (2015). "Review of national and international initiatives on books and book publishers assessment". El profesional de la información, v. 24, n. 6, pp. 705-716. https://doi.org/10.3145/epi.2015.nov.02

Giménez-Toledo, Elea; Román-Román, Adelaida (2009). "Assessment of humanities and social sciences monographs through their publishers: A review and a study towards a model of evaluation. Research evaluation, v. 18, n. 3, pp. 201-213. https://doi.org/10.3152/095820209X471986

Giménez-Toledo, Elea; Tejada-Artigas, Carlos; Mañana-Rodríguez, Jorge (2013). “Evaluation of scientific books' publishers in social sciences and humanities: Results of a survey". Research evaluation, v. 22, n. 1, pp. 64-77.

https://doi.org/10.1093/reseval/rvs036

Kulczycki, Emanuel (2018). "The diversity of monographs: Changing landscape of book evaluation in Poland". Aslib journal of information management, v. 70, n. 6, pp. 608-622.

https://doi.org/10.1108/AJIM-03-2018-0062

Kulczycki, Emanuel; Engels, Tim C. E.; Pölönen, Janne; Bruun, Kasper; Dušková, Marta; Guns, Raf; Nowotniak, Robert; Petr, Michal; Sivertsen, Gunnar; Starčič, Andreja-Istenič; Zuccala, Alesia (2018). "Publication patterns in the social sciences and humanities: Evidence from eight European countries". Scientometrics, v. 116, n. 1, pp. 463-486.

https://doi.org/10.1007/s11192-018-2711-0

Mañana-Rodríguez, Jorge; Pölönen, Janne (2018). "Scholarly book publishers' ratings and lists in Finland and Spain". Aslib journal of information management, v. 70, n. 6, pp. 643-659.

https://doi.org/10.1108/AJIM-05-2018-0111

Marques-Neto, José-Castilho; Rosa, Flávia-Garcia (2010). "Editoras universitárias: academia ou mercado? Reflexões sobre um falso problema”. En: Bragança, Aníbal; Abreu, Márcia (org.). Impresso no Brasil. Dois séculos de livros brasileiros. São Paulo: Unesp, pp. 331-348. ISBN: 9788539300518

Menandro, Paulo-Rogério-Meira; Yamamoto, Oswaldo-Hajime; Tourinho, Emmanuel-Zagury; Bastos, Antônio-Virgílio-Bittencourt (2011). "Livros à mão cheia: O livro como veículo de produção acadêmica”. Psicologia USP, v. 22, n. 2, pp. 367-386. http://www.scielo.br/pdf/pusp/v22n2/v22n2a05.pdf

Miranda, Elaine-Cristina; Mugnaini, Rogerio (2014). "Estudo exploratório dos critérios de classificação de livros nas áreas de Ciências Sociais Aplicadas, Humanas e Linguística, Letras \& Artes". En: Encontro brasileiro de bibliometria e cientometria, 4. Recife: UFPE.

http://www.brapci.inf.br/index.php/res/download/55572

Molas-Gallart, Jordi (2015). "Research evaluation and the assessment of public value". Arts and humanities in higher education, v. 14, n. 1, pp. 111-126.

https://doi.org/10.1177/1474022214534381

Nederhof, Anton J. (2006). "Bibliometric monitoring of research performance in the social sciences and the humanities: A review". Scientometrics, v. 66, n. 1, pp. 81-100.

https://doi.org/10.1007/s11192-006-0007-2

Paolinelli, Sônia-Maria-Rezende; Gaydeczka, Beatriz; Anjo, Luiz-Fernando-Resende-dos-Santos (2019). "As editoras universitárias no contexto da educação superior pública brasileira". Perspectivas em ciência da informação, v. 24, n. 1, pp. 25-38.

https://doi.org/10.1590/1981-5344/3134

Rodrigues, Kátia-de-Oliveira; Rosa, Flávia-Goulart; Oliveira, Marlene; Barros, Susane (2018). "O livro no sistema de avaliação da Capes". SciELO 20 years repository, pp. 1-23.

https://repository.scielo20.org/documents/article/view/129

Sivertsen, Gunnar (2014). "Scholarly publication patterns in the social sciences and humanities and their coverage in Scopus and Web of Science". En: Noyons, Ed. Proceedings of the science and technology indicators conference. Leiden: Universiteit, pp. 598-604, ISBN: 9789081752718

https://zenodo.org/record/2560399\#.XSReko-E5RA 